\title{
NEW EXISTENCE RESULTS FOR THE MEAN FIELD EQUATION ON COMPACT SURFACES VIA DEGREE THEORY
}

\author{
ALEKS JEVNIKAR
}

\begin{abstract}
We consider the following class of equations with exponential nonlinearities on a closed surface $\Sigma$ :

$$
-\Delta u=\rho_{1}\left(\frac{h e^{u}}{\int_{\Sigma} h e^{u} d V_{g}}-\frac{1}{|\Sigma|}\right)-\rho_{2}\left(\frac{h e^{-u}}{\int_{\Sigma} h e^{-u} d V_{g}}-\frac{1}{|\Sigma|}\right),
$$

which arises as the mean field equation of the equilibrium turbulence with arbitrarily signed vortices. Here $h$ is a smooth positive function and $\rho_{1}, \rho_{2}$ two positive parameters. By considering the parity of the Leray-Schauder degree associated to the problem, we prove solvability for $\rho_{i} \in(8 \pi k, 8 \pi(k+1)), k \in \mathbb{N}$. Our theorem provides a new existence result in the case when the underlying manifold is a sphere and gives a completely new proof for other known results.
\end{abstract}

\section{INTRODUCTION}

We are concerned with the equation

$$
-\Delta u=\rho_{1}\left(\frac{h e^{u}}{\int_{\Sigma} h e^{u} d V_{g}}-\frac{1}{|\Sigma|}\right)-\rho_{2}\left(\frac{h e^{-u}}{\int_{\Sigma} h e^{-u} d V_{g}}-\frac{1}{|\Sigma|}\right) \text { on } \Sigma,
$$

where $\Delta=\Delta_{g}$ is the Laplace-Beltrami operator, $\rho_{1}, \rho_{2}$ are two non-negative parameters, $h: \Sigma \rightarrow \mathbb{R}$ is a smooth positive function and $\Sigma$ is a compact orientable surface without boundary with Riemannian metric $g$ and total volume $|\Sigma|$. For the sake of simplicity, we will assume throughout this work that $|\Sigma|=1$, which is no loss of generality thanks to a trivial rescaling argument.

Problem (1) plays an important role in mathematical physics as a mean field equation for the equilibrium turbulence with arbitrarily signed vortices. It was first obtained by Joyce and Montgomery [16] and by Pointin and Lundgren 29] by means of different statistical arguments. Later, several authors adopted this model; we refer for example to [9], 22], 25, 27] and the references therein. The case $\rho_{1}=\rho_{2}$ is also related to the study of constant mean curvature surfaces, see [33, 34.

Before describing the main features of the problem and the known results, let us first consider the case $\rho_{2}=0$, namely the following standard Liouville-type equation:

$$
-\Delta u=\rho\left(\frac{h e^{u}}{\int_{\Sigma} h e^{u} d V_{g}}-1\right) .
$$

Equation (2) appears in conformal geometry in the problem of finding a conformal metric for which the Gauss curvature is a prescribed function on $\Sigma$, see [1], [6], [7,

2000 Mathematics Subject Classification. 35J20, 35J61, 35R01.

Key words and phrases. Geometric PDEs, Leray-Schauder degree, Mean field equation. 
20], 30. Indeed, setting $\tilde{g}=e^{2 v} g$, the Laplace-Beltrami operator for the deformed metric is given by $\Delta_{\tilde{g}}=e^{-2 v} \Delta_{g}$ and the change of the Gauss curvature is ruled by

$$
-\Delta_{g} v=K_{\tilde{g}} e^{2 v}-K_{g}
$$

where $K_{g}$ and $K_{\tilde{g}}$ are the Gauss curvatures of $(\Sigma, g)$ and of $(\Sigma, \tilde{g})$ respectively. Problem (2) also arises in mathematical physics as a mean field equation of Euler flows, see [5, 17. The literature on (2) is broad, and there are many results regarding existence, blow-up analysis, compactness of solutions, etc, see [10], 11], 24], 31.

As many geometric problems, also (2) carries a lack of compactness, as its solutions might blow-up. It was proved in [4, [19] and 21] that a quantization phenomenon occurs in this case. More precisely, taking a blow-up point $p$ for a sequence $\left(u_{n}\right)_{n}$ of solutions, we have

$$
\lim _{r \rightarrow 0} \lim _{n \rightarrow+\infty} \int_{B_{r}(p)} h e^{u_{n}} d V_{g}=8 \pi .
$$

Roughly speaking, each blow-up point carries a quantized local mass. Moreover, the limit profile of solutions becomes close to a bubble, namely a function $U_{\lambda, p}$ defined as

$$
U_{\lambda, p}(y)=\log \left(\frac{4 \lambda}{\left(1+\lambda d(p, y)^{2}\right)^{2}}\right),
$$

where $y \in \Sigma, d(p, y)$ stands for the geodesic distance and $\lambda$ is a large parameter. This limit function can be viewed as the logarithm of the conformal factor of the stereographic projection from $S^{2}$ onto $\mathbb{R}^{2}$, composed with a dilation.

Combining the local quantization (3) with some further analysis, see for example [3, 4], we have that the set of solutions to (2) is uniformly bounded in $C^{2, \alpha}$, for any fixed $\alpha \in(0,1)$, provided $\rho \notin 8 \pi \mathbb{N}$. It follows that one can define the LeraySchauder degree associated to problem (2) with $\rho \in(8 k \pi, 8(k+1) \pi), k \in \mathbb{N}$. In [19] it was shown that the degree is 1 when $\rho<8 \pi$. By the homotopic invariance of the degree, it is easy to see that the same is independent of the function $h$, the metric of $\Sigma$ and it is constant on each interval $(8 k \pi, 8(k+1) \pi)$. In fact it depends only on $k \in \mathbb{N}$ and the topological structure of $\Sigma$, as was proved in $[8$, where the authors provide the degree-counting formula

$$
\operatorname{deg}(\rho)=\frac{1}{k !}(-\chi(\Sigma)+1) \cdots(-\chi(\Sigma)+k),
$$

where $\chi(\Sigma)$ denotes the Euler characteristic of $\Sigma$. The proof of this result is carried out by analyzing the jump values of the degree after $\rho$ crossing the critical thresholds. Later, this result was rephrased in 223] with a Morse theory point of view.

On the other hand, in the general case when $\rho_{2} \neq 0$, namely for equation (11), there are fewer results: for example the refined blow-up analysis of solutions of (1) is not yet fully developed. Nevertheless, it was proven in 15] that the blow-up phenomenon yields a quantization property; for a blow-up point $p$ and a sequence $\left(u_{n}\right)_{n}$ of solutions relatively to $\left(\rho_{1, n}, \rho_{2, n}\right)$ the authors obtained

$$
\lim _{r \rightarrow 0} \lim _{n \rightarrow+\infty} \rho_{1, n} \frac{\int_{B_{r}(p)} h e^{u_{n}} d V_{g}}{\int_{\Sigma} h e^{u_{n}} d V_{g}} \in 8 \pi \mathbb{N}, \quad \lim _{r \rightarrow 0} \lim _{n \rightarrow+\infty} \rho_{2, n} \frac{\int_{B_{r}(p)} h e^{-u_{n}} d V_{g}}{\int_{\Sigma} h e^{-u_{n}} d V_{g}} \in 8 \pi \mathbb{N} .
$$


Moreover, the case of positive multiples of $8 \pi$ may indeed occur, see [12] and [13. The latter volume quantization implies that the set of solutions is compact for $\left(\rho_{1}, \rho_{2}\right) \notin(8 \pi \mathbb{N} \times \mathbb{R}) \cup(\mathbb{R} \cup 8 \pi \mathbb{N})$.

It follows that, as before, the degree associated to (10) can still be defined outside this set of parameters. However, this strategy has not been yet investigated and the existence results mostly rely on a variational approach.

Let us now briefly discuss the history of the problem and the previous results. First of all, the case $\rho_{1}, \rho_{2}<8 \pi$ was considered in 28. Ohtsuka and Suzuki proved that in this regime the associated energy functional is bounded from below and coercive and therefore solutions can be found as global minima. If $\rho_{i}>8 \pi$ for some $i=1,2$, then the problem becomes subtler as the functional is not bounded and a minimization technique is not possible any more. The first result in this direction is given in [15] for $\rho_{1} \in(8 \pi, 16 \pi)$ and $\rho_{2}<8 \pi$. Via a blow-up analysis Jost et al. proved the existence of solutions on a smooth, bounded, non-simply connected domain in $\mathbb{R}^{2}$ with homogeneous Dirichlet boundary condition. Later, in [32], Zhou generalized this result to any compact surface without boundary by reducing the problem to the one with $\rho_{2}=0$ and applying min-max theory.

The next step was made in [14, where for the first time a doubly supercritical regime is considered, namely $\rho_{i} \in(8 \pi, 16 \pi)$ for $i=1,2$. The existence of solutions is proved using a min-max scheme based on a detailed description of the low sublevels of the energy functional. Finally, this was generalized in $[2$ for a generic choice of the parameters $\rho_{1} \in(8 k \pi, 8(k+1) \pi)$ and $\rho_{2} \in(8 l \pi, 8(l+1) \pi), k, l \in \mathbb{N}$, under the assumption that $\Sigma$ has positive genus.

\section{THE MAIN RESULT}

We attack the problem with a different point of view and for the first time we analyze the associated Leray-Schauder degree. This is done in the spirit of [26], where a Toda system of Liouville equations arising from Chern-Simons theory was analyzed. More precisely, we study its parity and we observe that when both parameters stay in the same interval, i.e. $\rho_{i} \in(8 k \pi, 8(k+1) \pi), k \in \mathbb{N}$ for $i=1,2$, the degree is always odd. The main result is the following.

Theorem 2.1. Let $h>0$ be a smooth function and suppose $\rho_{i} \in(8 k \pi, 8(k+1) \pi)$, $k \in \mathbb{N}$ for $i=1,2$. Then problem (1) has a solution.

Observe that we recover the result of [14] and some cases of [2]: when $\Sigma$ is homeomorphic to $S^{2}$ the above theorem yields a new existence result.

Proof. For some $\alpha \in(0,1)$ let $C_{0}^{2, \alpha}(\Sigma)$ be the class of $C^{2, \alpha}$ functions with zero average. Consider now the mapping $T: C_{0}^{2, \alpha}(\Sigma) \rightarrow C_{0}^{2, \alpha}(\Sigma)$ defined by

$$
T(u)=(-\Delta)^{-1}\left(\rho_{1}\left(\frac{h e^{u}}{\int_{\Sigma} h e^{u} d V_{g}}-1\right)-\rho_{2}\left(\frac{h e^{-u}}{\int_{\Sigma} h e^{-u} d V_{g}}-1\right)\right),
$$

where $(-\Delta)^{-1} f, f \in C^{\alpha}(\Sigma)$, is intended as the solution $v$, with zero average, of the problem $-\Delta v=f$, which is unique. We are concerned with the map $\Psi=I d-T$ and the solutions of equation (1) will correspond to zeros of $\Psi$.

Clearly, by elliptic regularity theory the operator $T$ is compact. Moreover, the set of the solutions is compact for parameters $\left(\rho_{1}, \rho_{2}\right) \notin(8 \pi \mathbb{N} \times \mathbb{R}) \cup(\mathbb{R} \times 8 \pi \mathbb{N})$. 
Therefore, we can consider the associated degree $\operatorname{deg}\left(\Psi_{\left(\rho_{1}, \rho_{2}\right)}, B_{r}(0), 0\right)$ which is well-defined for $r$ sufficiently large.

Consider now $\rho_{i} \in(8 k \pi, 8(k+1) \pi), k \in \mathbb{N}$ for $i=1$, 2. Letting $\rho=\frac{1}{2}\left(\rho_{1}+\rho_{2}\right)$, we perform the following homotopy which takes place in a connected component of $\mathbb{R}^{2} \backslash((8 \pi \mathbb{N} \times \mathbb{R}) \cup(\mathbb{R} \times 8 \pi \mathbb{N})):$

$$
\mathfrak{h}(t)=(1-t)\left(\rho_{1}, \rho_{2}\right)+t(\rho, \rho) .
$$

From the fact that the degree is constant along homotopies we obtain that

$$
\operatorname{deg}\left(\Psi_{\left(\rho_{1}, \rho_{2}\right)}, B_{r}(0), 0\right)=\operatorname{deg}\left(\Psi_{(\rho, \rho)}, B_{r}(0), 0\right) .
$$

Observe now that by the structure of $T$ we deduce

$$
\Psi_{(\rho, \rho)}(-u)=-\Psi_{(\rho, \rho)}(u) .
$$

Therefore, we conclude that $\Psi_{(\rho, \rho)}$ is an odd operator. By the Borsuk theorem, see [18, it follows that the associated degree is odd an hence non zero. This guarantees us the existence of a solution to equation (11).

\section{Acknowledgements}

The author would like to thank Professor Andrea Malchiodi for his kind help in preparing this paper.

The author is supported by the PRIN project Variational and perturbative aspects of nonlinear differential problems.

\section{REFERENCES}

[1] A. Bahri - J.M. Coron, The scalar curvature problem on the standard three dimensional sphere, J. Funct. Anal., 95 (1991), pp. 106-172.

[2] L. Battaglia - A. Jevnikar - A. Malchiodi - D. Ruiz, A general existence result for the Toda system on compact surfaces, preprint (2013), arXiv/http://arxiv.org/abs/1306.5404.

[3] L. Battaglia - G. Mancini, A note on compactness properties of singular Toda systems, preprint (2014), arXiv http://arxiv.org/abs/1410.4991.

[4] H. Brezis - F. Merle, Uniform estimates and blow-up behavior for solutions of $-\Delta u=$ $V(x) e^{u}$ in two dimensions, Comm. Partial Differential Equations, 16 (1991), pp. 12231253.

[5] E. Caglioti - P.L. Lions - C. Marchioro - M. Pulvirenti, A special class of stationary flows for two-dimensional Euler equations: a statistical mechanics description, Part I, Comm. Math. Phys., 143 (1992), pp. 501-525. Part II, Comm. Math. Phys., 174 (1995), pp. 229-260.

[6] S.Y.A. Chang - M.J. Gursky - P.C. Yang, The scalar curvature equation on 2- and 3spheres, Calc. Var. and Partial Diff. Eq., 1 (1993), pp. 205-229.

[7] S.Y.A. Chang - P.C. Yang, Prescribing Gaussian curvature on $S^{2}$, Acta Math., 159 (1987), pp. 215-259.

[8] C.C. Chen - C.S. Lin, Topological degree for a mean field equation on Riemann surfaces, Comm. Pure Appl. Math., 56 (2003), pp. 1667-1727.

[9] A.J. Chorin, Vorticity and Turbulence, Springer, New York, 1994.

[10] W. Ding - J. Jost - J. Li - G. Wang, Existence results for mean field equations, Ann. Inst. Henri Poincaré, Anal. Non Linèaire, 16 (1999), pp. 653-666.

[11] Z. Djadli, Existence result for the mean field problem on Riemann surfaces of all genus, Comm. Contemp. Math., 10 (2008), pp. 205-220.

[12] P. Esposito - J. Wei, Non-simple blow-up solutions for the Neumann two-dimensional sinh-Gordon equation, Calc. Var. Partial Differential Equations, 34 (2009), pp. 341-375.

[13] M. Grossi - A. Pistoia, Multiple blow-up phenomena for the sinh-poisson equation, Archive Rat. Mech. and Anal., 209 (2013), pp. 287-320. 
[14] A. Jevnikar, An existence result for the mean-field equation on compact surfaces in a doubly supercritical regime, Proc. Royal Soc. Edinburgh A, 143 (2013), pp. 1021-1045.

[15] J. Jost - G. Wang - D. Ye - C. Zhou, The blow up analysis of solutions of the elliptic sinh-Gordon equation, Calc. Var. Partial Differential Equations, 31 (2008), pp. 263-276.

[16] G. Joyce - D. Montgomery, Negative temperature states for the two-dimensional guidingcentre plasma, J. Plasma Phys., 10 (1973), pp. 107-121.

[17] M. Kiessling, Statistical mechanics of classical particles with logarithmic interactions, Comm. Pure Appl. Math., 46 (1993), pp. 27-56.

[18] W.T. Kyner, A Generalization of the Borsuk and Borsuk-Ulam Theorems, Proc. Amer. Math. Soc., 7 (1956), pp. 1117-1119.

[19] Y.Y. Li, Harnack type inequality: the method of moving planes, Comm. Math. Phys., 200 (1999), pp. 421-444.

[20] Y.Y. Li, Prescribing scalar curvature on $S^{n}$ and related topics, Part I, Journal of Differential Equations, 120 (1995), pp. 319-410. Part II, Existence and compactness, Comm. Pure Appl. Math., 49 (1996), pp. 437-477.

[21] Y.Y. Li - I. Shafrir, Blow-up analysis for solutions of $-\Delta u=V e^{u}$ in dimension two, Indiana Univ. Math. J., 43 (1994), pp. 1255-1270.

[22] P.L. Lions, On Euler Equations and Statistical Physics, Scuola Normale Superiore, Pisa, 1997.

[23] A. Malchiodi, Morse theory and a scalar field equation on compact surfaces, Adv. Differential Equations, 13 (2008), pp. 1109-1129.

[24] A. Malchiodi, Topological methods for an elliptic equation with exponential nonlinearities, Discrete Contin. Dyn. Syst., 21 (2008), pp. 277-294.

[25] C. Marchioro - M. Pulvirenti, Mathematical theory of incompressible nonviscous fluids, Springer, New York, 1994.

[26] A. Malchiodi - D. Ruiz, On the Leray-Schauder degree of the Toda system on compact surfaces, preprint (2014), arXiv http://arxiv.org/abs/1311.7375.

[27] P.K. Newton, The N-Vortex Problem: Analytical Techniques, Springer, New York, 2001.

[28] H. Ohtsuka - T. Suzuki, Mean field equation for the equilibrium turbulence and a related functional inequality, Adv. Differential Equations, 11 (2006), pp. 281-304.

[29] Y.B. Pointin - T.S. Lundgren, Statistical mechanics of two-dimensional vortices in a bounded container, Phys. Fluids, 19 (1976), pp. 1459-1470.

[30] R. Schoen - D. Zhang, Prescribed scalar curvature on the n-sphere, Calc. Var., 4 (1996), pp. $1-25$.

[31] G. Tarantello, Analytical, geometrical and topological aspects of a class of mean field equations on surfaces, Discrete Contin. Dyn. Syst., 28 (2010), pp. 931-973.

[32] C. Zhou, Existence of solution for mean-field equation for the equilibrium turbulence, Nonlin. Analysis, 69 (2008), pp. 2541-2552.

[33] H.C. Wente, Large solutions to the volume constrained Plateau problem, Arch. Rational Mech. Anal., 75 (1980/81), pp. 59-77.

[34] H.C. Wente, Counterexample to a conjecture of H. Hopf, Pacific J. Math., 121 (1986), pp. 193-243.

SISSA, Via Bonomea 265, 34136 Trieste (ItAly).

E-mail address: ajevnika@sissa.it 